Sportwiss 2013 - 43:235-238

DOI 10.1007/s12662-013-0312-x

Online publiziert: 22. Oktober 2013

๑) Springer-Verlag Berlin Heidelberg 2013
Ralf Brand $^{1} \cdot$ Claudia Voelcker-Rehage $^{2} \cdot$ Doris Superina $^{1}$

${ }^{1}$ Department für Sport- und Gesundheitswissenschaften, Universität Potsdam, Potsdam, Deutschland

2Jacobs Center für Lebenslanges Lernen, Jacobs University Bremen, Bremen, Deutschland

\title{
Was leistet die Sportwissenschaft?
}

\author{
Vom Machen und Mitmachen \\ an einer Zeitschrift
}

Wissenschaft wird durch ihre Publikation hervorgebracht. Wissenschaftliche Zeitschriften dienen dazu, den aktuellen Erkenntnisstand wissenschaftlicher Fächer aufzugreifen, zu repräsentieren und ihn fortzuschreiben.

Sicherlich in diesem Sinne wurde 1971 die Sportwissenschaft von Ommo Grupe gegründet. Mit Blick auf den aus seiner Sicht damals „ohne Zweifel niedrigen Standard sportwissenschaftlicher Forschung in der Bundesrepublik" (Grupe, 1971, S. 8), befürchtete er, es könne sich vielleicht um ein „zu früh geborenes Kind einer wissenschaftlichen Disziplin“ (ebd., S. 7) handeln. Die Sorge erwies sich als unbegründet. Die Zeitschrift erblühte und hat sicher zur Entwicklung unseres Faches Sportwissenschaft erheblich beigetragen.

Über Entwicklungen in den ersten 20 Jahrgängen der Sportwissenschaft bilanziert Digel (1992a, b). Zur Jahrhundertwende folgt eine weitere Bewertung. Im Beitrag zu einigen strukturellen Merkmalen der Zeitschrift hält Digel (2000a, S. 19) u. a. fest, dass die Sportwissenschaft zwar ein Publikationsorgan darstelle, das für Offenheit und Vielfalt hinsichtlich sportwissenschaftlicher Veröffentlichungen stehe, jedoch sei einigen Disziplinen, insbesondere der Trainingswissenschaft und der Sportmedizin zu wünschen, dass sich ihre Beitragsanteile kräftig erhöhen. Die genauere Betrachtung von Autorenschaften und Autorennetzwerken (Digel, 2000b, S. 346) führt ihn zu verschiedenen kritischen Bemerkungen über das „Netzwerk Tübingen“, welches die Inhalte der Zeitschrift über weite Strecken domi- niere, jedoch gleichermaßen zu der Einschätzung, dass die „besondere Qualität“ der Sportwissenschaft eben auch herausragenden Sportwissenschaftlern dieses Netzwerks geschuldet sei. Mahnend weist er schließlich darauf hin, dass die internationale Kommunikation von Erkenntnissen nur unzureichend gelänge. Der schon von Grupe (1971, S. 18) formulierte Anspruch, dass Deutsch nicht die einzige Sprache der Zeitschrift bleiben möge, sondern englische und ggf. französische Beiträge im Original die Zeitschrift bereichern sollten, wurde bis zur Jahrtausendwende nur in den Anfangsjahren erfüllt (1971-1975: 7,4\% englischsprachige Beiträge) und ab 1996 gar nicht mehr (bis 1996-1999: 0\%), wie Digel (2000b) berichtet.

Im Jahr 2013 wagen wir einen weiteren Versuch der Bilanzierung, besser, der Bestimmung eines Zwischenstandes. Wieso?

Nun, vielleicht befindet sich die Sportwissenschaft derzeit an einem Scheidepunkt. Anfang des Jahres 2009 wechselte die Zeitschrift vom Hofmann-Verlag (Schorndorf) zum international orientierten und hoch renommierten Verlagshaus Springer (Heidelberg). Für die Sportwissenschaft sind die damit verbundenen Möglichkeiten, insbesondere die im Online-Publikationswesen deutlich verbesserte internationale Sichtbarkeit, Chance und Herausforderung zugleich. Notwendigerweise steht die Sportwissenschaft damit aber auch im (direkten) Vergleich zu den Zeitschriften anderer Fachdisziplinen. Sie ist gefordert, und wir sind entschlossen, ihre Leistungsfähigkeit im Ver- gleich zu anderen Fachorganen nachzuweisen. So haben sich einige wichtige Leistungsindizes unserer Zeitschrift seit dem Wechsel zum Springer-Verlag stetig verbessert. Insbesondere diese Entwicklung soll im Nachfolgenden kurz skizziert werden.

Allerdings, und damit sei noch einmal unterstrichen, was Grupe (1971, S. 15) und Digel (2000b, S. 326) schon lange vor uns festgehalten haben: Die Sportwissenschaft kann nur so gut sein, wie die wissenschaftlichen Beiträge, die ihr zur Publikation angeboten werden!

Mit den folgenden Darstellungen verbindet sich deshalb vor allem der Appell an die Kolleginnen und Kollegen Sportwissenschaftler, die willens und fähig sind, der Zeitschrift und gleichermaßen dem Fach Sportwissenschaft, durch die Berichtlegung ihrer wissenschaftlichen Erkenntnisse nach vorne zu helfen, noch in viel größerer Zahl hervorragende Beiträge einzureichen! Denn der Widerhall, den in der Sportwissenschaft publizierte Fachartikel in den vergangenen Jahren gefunden haben, ist bescheiden. Ob die Sportwissenschaft die Bedeutung zurückgewinnt, welche sie in früheren Jahren schon einmal für unser Fach hatte oder ob sie weiter einbüßt, wird sich in den nächsten 3 Jahren entscheiden.

Einige der vorliegenden Darstellungen entstammen der Bachelor-Arbeit von Doris Superina, die ihr Studium im August 2013 an der Universität Potsdam mit Erfolg abschloss. 


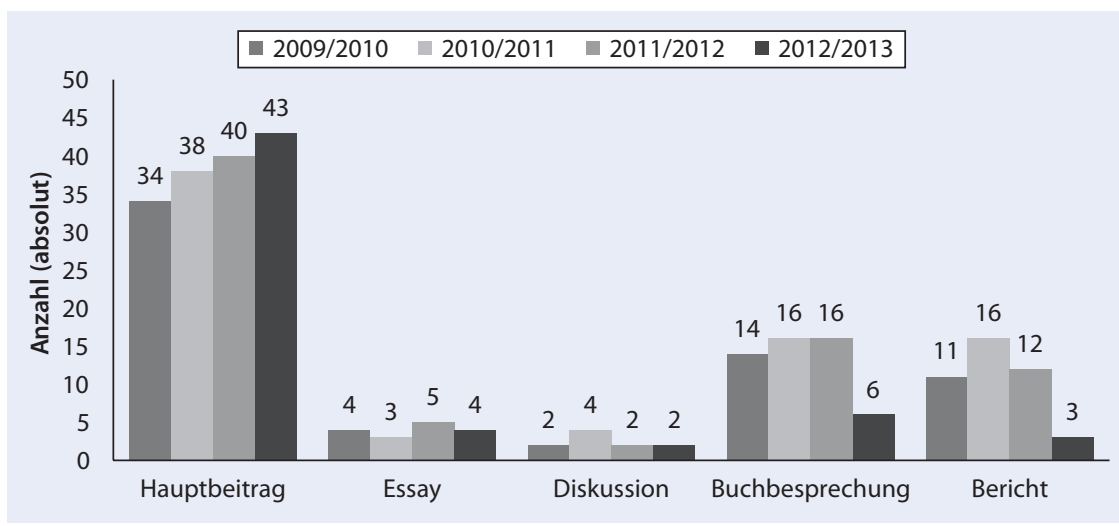

Abb. 1 \ Anzahl der eingereichten Manuskripte

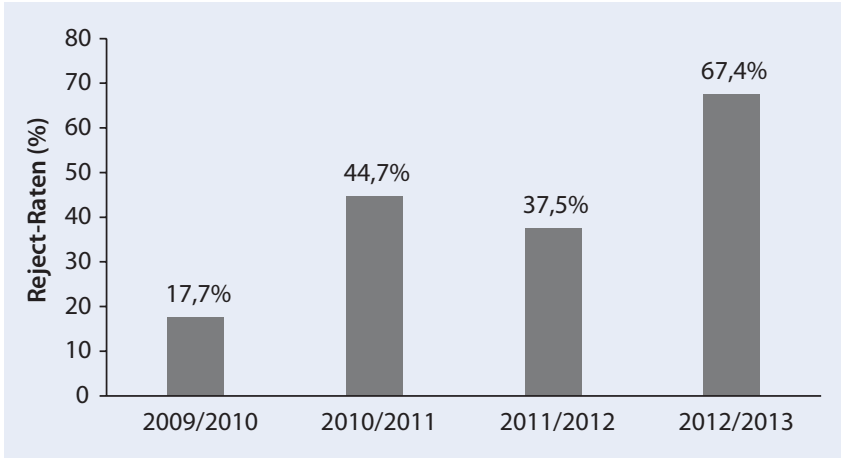

Abb. $2 \varangle$ Reject-Raten

\section{Analyse der aktuellen Publikationsleistung}

Die nachfolgenden Auswertungen beziehen sich auf den Zeitraum Mai 2009 bis April 2013. Die Entwicklung wird in vier etwa gleich großen, disjunkten Zeitabschnitten betrachtet. Diese sind in allen Abbildungen wie folgt benannt: Der Datenpunkt 2009/2010 umfasst den Zeitraum Mai 2009 bis März 2010, 2010/2011 beschreibt die Monate April 2010 bis März 2011, 2011/2012 die Zeit von April 2011 bis März 2012, und 2012/2013 die Zeit April 2012 bis März 2013. Wichtigster Grund für diese Zeiteinteilung ist die mit dem Wechsel zum Springer-Verlag verbundene (und seit Mai 2009 technisch vollzogene) Umstellung des Begutachtungs- und Publikationsverfahrens der Zeitschrift auf ein onlinegestütztes Datenbanksystem (Editorial Manager). Die Wahl des Betrachtungsendpunktes (zum 1. April 2013) ergibt sich aus der Überlegung heraus, dass damit das Wirken der zum 1. April 2012 neu berufenen Herausgeberrunde zwischenbilanziert werden kann.
Anzahl eingereichter Beiträge. Auf den Internetseiten der dvs sind aktuell 67 sportwissenschaftliche Hochschuleinrichtungen in Deutschland gelistet. Deren Mitarbeiterinnen und Mitarbeiter, und sicherlich noch einige darüber hinaus, können als Personengrundstock für die Teilhabe an der Zeitschrift betrachtet werden.

In den betrachteten 4 Jahren wurden insgesamt 129 Hauptbeiträge (und in deutlich geringeren Zahlen andere Beitragsarten) zur Begutachtung eingereicht (- Abb. 1). Die Teilhabe der an sportwissenschaftlichen Hochschuleinrichtungen arbeitenden Wissenschaftler und Wissenschaftlerinnen an ihrer Zeitschrift, kann damit als sehr gering bezeichnet werden.

Reject-Raten. Vor der Veröffentlichung von Beiträgen, insbesondere Hauptbeiträgen, durchlaufen diese einen Begutachtungsprozess im Blind-peer-reviewVerfahren. Mindestens zwei zum jeweiligen Thema fachlich ausgewiesene Experten empfehlen den Herausgebern entweder die Publikation des eingereichten Beitrags, die Zurückweisung zur Überarbeitung nach Gutachterempfehlungen („minor revision, „major revision“, „reject and resubmit") oder die Ablehnung des Manuskripts („reject“). Dieses System der Kontrolle soll eine möglichst hohe Qualität der publizierten Beiträge sichern. Eine Betrachtung der Reject-Raten erlaubt Rückschlüsse auf die resultierende Qualität und das Qualitätsmanagement einer wissenschaftlichen Zeitschrift (- Abb. 2).

Die Daten lassen verschiedene Schlüsse zu. Einerseits könnte die Qualität der Beiträge zuletzt stark abgenommen haben, so dass deutlich mehr Artikel zurückgewiesen werden mussten. Andererseits mag aber auch zutreffen, dass sich Qualitätsansprüche verändert haben. Unser Ziel im Herausgebergremium war und ist eine bedachte, aber konsequente Anhebung der Qualitätsmaßstäbe, an denen sich zu publizierende Hauptbeiträge messen sollen (Brand \& Voelcker-Rehage, 2013). Die Daten lassen also auch den Schluss zu, dass die Zeitschrift leistungsstärker aufgestellt wurde. Qualitativ hochwertige und für ein Forschungsfeld wichtige Ergebnisse werden von anderen Sportwissenschaftlerinnen und Sportwissenschaftlern eher aufgegriffen (und z. B. in weiterführenden Arbeiten zitiert) als weniger hochwertige. Gewinnt eine Zeitschrift das Ansehen, dass in ihr vorwiegend hervorragende Arbeiten veröffentlicht werden, dann steigert dies die Attraktivität der Zeitschrift und sollte zu einer vergrößerten Teilhabe von Wissenschaftlerinnen und Wissenschaftlern führen.

\section{Sportwissenschaftliche Fachberei-} che. Die Sportwissenschaft verfolgt den Anspruch, möglichst Beiträge aus den 4 Fachbereichen Geisteswissenschaften (z. B. Sportpädagogik, Sportgeschichte), Sozialwissenschaften (z. B. Sportsoziologie, Sportökonomie und -management), Verhaltenswissenschaften (z. B. Sportpsychologie) und Medizin-/Naturwissenschaften (z. B. Trainings- und Bewegungswissenschaft, Biomechanik, Sportmedizin) zu publizieren (• Abb. 3).

Die Verteilung in $\bullet$ Abb. 3 zeigt, dass sich die Teilhabe durch die 4 Fachbereiche in den betrachteten 4 Jahresabschnitten dynamisch entwickelte. Ob sich der in den Daten sichtbare Trend festigt, der da- 


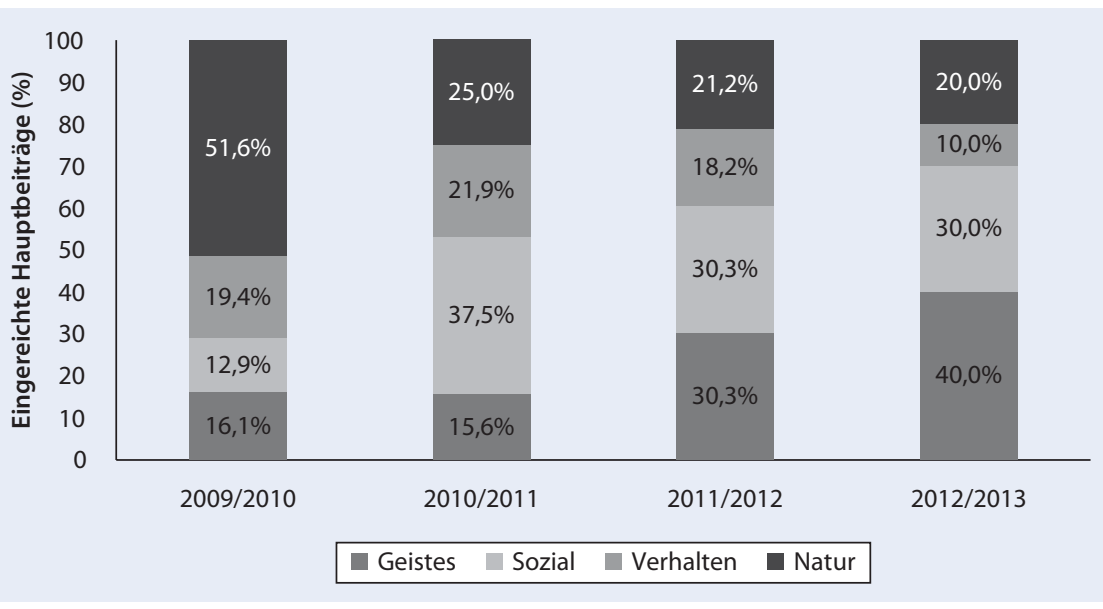

Abb. $3 \Delta$ Eingereichte Hauptbeiträge nach Fachbereichen

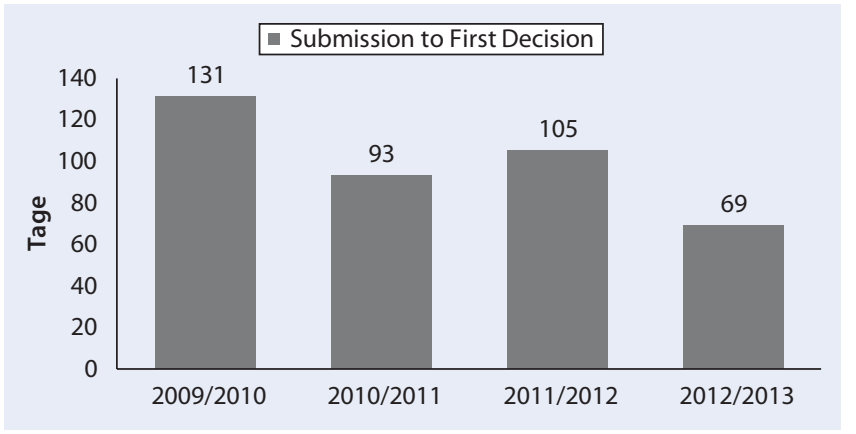

rauf hindeutet, dass der Sportwissenschaft in den vergangenen Jahren insbesondere immer weniger verhaltenswissenschaftliche und medizinisch-/naturwissenschaftliche Beiträge angeboten (!) werden, dürfte eine der spannenden Fragen sein, die wir in den kommenden Jahren im Auge behalten müssen.

Englischsprachige Beiträge. In den Jahreszeiträumen 2009/2010, 2010/2011 und 2011/2012 sind vier englischsprachige Beiträge erschienen. Im Zeitraum 2012/2013 kam ein einziger hinzu (mit Blick auf die aktuellsten Entwicklungen ist allerdings festzuhalten, dass in allen 3 Heftausgaben, die nach April 2013 publiziert wurden, jeweils ein englischsprachiger Beitrag ist). Der seit jeher bestehende Selbstanspruch der Sportwissenschaft, die internationale Kommunikation sportwissenschaftlicher Erkenntnisse, lässt sich diesen Zahlen zufolge aktuell nicht einlösen.

Anzahl der Zitationen. Die um die Berechnung von Impact-Faktoren ent-
Abb. $4<$ Zeitdauer zwischen Ersteinreichung und erster Entscheidung durch Editor

Sportwissenschaft in den kommenden Jahren entscheidet.

Schnelligkeit des Begutachtungsprozesses. Zeitschriftenpublikationen haben z. B. gegenüber Buchpublikationen den Vorteil, dass wissenschaftliche Erkenntnisse schnell, d. h. binnen weniger Monate nach Erlangen der Ergebnisse, publiziert werden können. Ein Zeichen der Leistungsfähigkeit wissenschaftlicher Zeitschriften besteht dementsprechend darin, dass die Zeitspanne, in welcher ein Manuskript bei den Herausgebern und/ oder Begutachtenden verbleibt, möglichst kurz ist (die Zeit zwischen Ersteinreichung des Manuskripts und Publikation ist demgegenüber nicht aussagekräftig, weil sich in diese auch die Bearbeitungsschnelligkeit der Autoren mischt). In - Abb. 4 wird die Entwicklung der Sportwissenschaft in den vergangenen 4 Jahren illustriert, mit Blick auf die Zeitdauer zwischen der Ersteinreichung des Beitrags und der ersten Entscheidung durch den betreuenden Herausgeber („submission to first decision“; d. h. „accept“, „minor revision“, „major revision“, „reject and resubmit“, „reject“).

Die mittlerweile für die Sportwissenschaft erreichte Bearbeitungsgeschwindigkeit ist im Vergleich zu manch großen internationalen Zeitschriften (oft werden 4 bis 6 Wochen erreicht) leider noch nicht konkurrenzfähig. Jedoch wird es ein Ziel der Zeitschrift bleiben, auch in dieser ganz objektiv fassbaren Leistungskennziffer noch stärker zu werden.

\section{Fazit}

Science und Scopus). Bei 16 bis 20 pubim Vergleich zu vielen anderen wissenschaftlichen Zeitschriften sehr wenig. Betrachtet man die Zitationshäufigkeiten in den drei vollständigen Kalenderjahren $(2010,2011,2012)$ innerhalb unseres Betrachtungszeitraums (von 5/2009 bis 3/2013), dann verteilen sich diese allerdings in ermutigender Weise: Im Jahr 2010 wurden vier, 2011 zwölf und 2012 immerhin 17 Zitationen erreicht. Ob dieser positive Trend das Ergebnis flüchtiger Schwankungen ist, ob er sich stabilisiert oder fortsetzen lässt, dürfte eine der Fragen sein, an der sich die Zukunft der
Wie lässt sich die Publikationsleistung der Sportwissenschaft nun also bewerten? In den vergangenen 4 Jahren und aktuell?

Unserer Einschätzung nach hat sich die Zeitschrift seit ihrem Wechsel zum Springer-Verlag in wesentlichen Kennzeichen, mitunter den beschriebenen, positiv weiterentwickelt. Jedoch erscheint es uns wichtig hervorzuheben, dass die Teilhabe der Kollegen Sportwissenschaftlerinnen und Sportwissenschaftler an unserer Zeitschrift noch nicht so ist, wie wir uns dies im Kreise der Herausgebenden idealerweise vorstellen würden. Mit 


\section{Editorial}

Blick auf das Zeitschriftenmanagement werden wir in unseren Bemühungen, die Sportwissenschaft weiter zu erstarken, keinesfalls nachlassen. Ob wir damit Erfolg haben werden, liegt nicht allein in unserer Hand. In diesem Sinne: Helfen Sie uns dabei. Wie wir schon zu Beginn unserer Amtszeit, in Heft 2/2012, im ersten Editorial geschrieben haben: „Auf geht's!" (Brand \& Voelcker-Rehage, 2012).

\section{Korrespondenzadresse}

\section{Prof. Dr. R. Brand}

Department für Sportund Gesundheitswissenschaften Universität Potsdam

Am Neuen Palais 10, 14469 Potsdam

ralf.brand@uni-potsdam.de

\section{Literatur}

Brand, R., \& Voelcker-Rehage, C. (2012). Auf geht's! Neubesetzung des Herausgeberkollegiums der Sportwissenschaft (Editorial). Sportwissenschaft, $42,80-82$.

Brand, R., \& Voelcker-Rehage, C. (2013). Zeit der Veränderung (Editorial). Sportwissenschaft, 43, 83-84.

Digel, H. (1992a). Sportwissenschaft in der Zeitschrift "Sportwissenschaft". Formale Strukturen und inhaltliche Merkmale. Sportwissenschaft, 22, 60-85.

Digel, H. (1992b). Zwanzig Jahre Zeitschrift „Sportwissenschaft". Autoren und personelle Netzwerke. Sportwissenschaft, 22, 440-466.

Digel, H. (2000a). Strukturelle Merkmale der Zeitschrift "Sportwissenschaft": Rückblick und Ausblick am Ende eines Jahrhunderts. Sportwissenschaft, 30, 1-19.

Digel, H. (2000b). Die Autorinnen und Autoren der Zeitschrift "Sportwissenschaft“. Rückblick auf 29 Jahrgänge. Sportwissenschaft, 30, 326-347.

Grupe, O. (1971). Einleitung in die "Sportwissenschaft" (oder: Über die Schwierigkeit eine neue Publikation zu planen) (Editorial). Sportwissenschaft, 1 , 7-18. 